OPEN ACCESS

Edited by:

Orly Lazarov,

University of Illinois at Chicago,

United States

Reviewed by:

Panteleimon Giannakopoulos, Université de Genève, Switzerland Taher Darreh-Shori,

Karolinska Institutet, Sweden

${ }^{*}$ Correspondence: Katherine J. Bangen kbangen@ucsd.edu

Received: 10 February 2017 Accepted: 24 May 2017 Published: 08 June 2017

Citation:

Bangen KJ, Clark AL, Edmonds EC, Evangelista ND, Werhane $M L$, Thomas KR, Locano LE, Tran M, Zlatar ZZ, Nation DA, Bondi MW and

Delano-Wood $L$ for the Alzheimer's Disease Neuroimaging Initiative (2017) Cerebral Blood Flow and Amyloid- $\beta$ Interact to Affect Memory Performance in Cognitively Normal Older Adults.

Front. Aging Neurosci. 9:181 doi: 10.3389/fnagi.2017.00181

\section{Cerebral Blood Flow and Amyloid- $\beta$ Interact to Affect Memory Performance in Cognitively Normal Older Adults}

\author{
Katherine J. Bangen ${ }^{1,2 *}$, Alexandra L. Clark ${ }^{3}$, Emily C. Edmonds ${ }^{1,2}$, \\ Nicole D. Evangelista', Madeleine L. Werhane ${ }^{3}$, Kelsey R. Thomas ${ }^{1,4}$, Lyzette E. Locano ${ }^{5}$, \\ My Tran ${ }^{5}$, Zvinka Z. Zlatar' ${ }^{2}$, Daniel A. Nation ${ }^{6}$, Mark W. Bondi ${ }^{2,4}$ and \\ Lisa Delano-Wood ${ }^{1,2}$ for the Alzheimer's Disease Neuroimaging Initiative ${ }^{\dagger}$
}

${ }^{1}$ Research Service, VA San Diego Healthcare System, San Diego, CA, United States, ${ }^{2}$ Department of Psychiatry, University of California, San Diego, La Jolla, CA, United States, ${ }^{3}$ San Diego State University, University of California, San Diego Joint Doctoral Program in Clinical Psychology, San Diego, CA, United States, ${ }^{4}$ Psychology Service, VA San Diego Healthcare System, San Diego, CA, United States, ${ }^{5}$ Department of Psychology, San Diego State University, San Diego, CA, United States, ${ }^{6}$ Department of Psychology, University of Southern California, Los Angeles, CA, United States

Cerebral blood flow (CBF) alterations and amyloid- $\beta$ (A $\beta$ ) accumulation have been independently linked to cognitive deficits in older adults at risk for dementia. Less is known about how $\mathrm{CBF}$ and $\mathrm{A} \beta$ may interact to affect cognition in cognitively normal older adults. Therefore, we examined potential statistical interactions between CBF and $A \beta$ status in regions typically affected in Alzheimer's disease (AD) within a sample of older adults from the Alzheimer's Disease Neuroimaging Initiative (ADNI) study. Sixtytwo cognitively normal participants (mean age $=72$ years) underwent neuroimaging and memory testing. Arterial spin labeling magnetic resonance imaging was used to quantify CBF and florbetapir PET amyloid imaging was used to measure A $\beta$ deposition. $A \beta$ status (i.e., positivity versus negativity) was determined based on established cutoffs (Landau et al., 2013). The Rey Auditory Verbal Learning Test was used to assess memory. Linear regression models adjusted for age, education, and sex, demonstrated significant interactions between $\mathrm{CBF}$ and $\mathrm{A} \beta$ status on memory performance. Among $A \beta$ positive older adults, there were significant negative associations between higher CBF in hippocampus, posterior cingulate, and precuneus and poorer memory performance. In contrast, among $A \beta$ negative older adults, there were no significant associations between CBF and cognition. Our findings extend previous CBF studies of dementia risk by reporting interactions between $A \beta$ status and CBF on memory performance in a sample of well-characterized, cognitively normal older adults. Results suggest that differential CBF-cognition associations can be identified in healthy, asymptomatic $A \beta$ positive older adults relative to $A \beta$ negative individuals. Associations between higher

\footnotetext{
${ }^{\dagger}$ Data used in preparation of this article were obtained from the Alzheimer's Disease Neuroimaging Initiative (ADNI) database (adni.loni.usc.edu). As such, the investigators within the ADNI contributed to the design and implementation of ADNI and/or provided data but did not participate in analysis or writing of this report. A complete listing of ADNI investigators can be found at: http://adni.loni.usc.edu/wp-content/uploads/how_to_apply/ADNI_Acknowledge ment_List.pdf
} 
CBF and poorer memory among $A \beta$ positive older adults may reflect a cellular and/or vascular compensatory response to pathologic processes whereby higher CBF is needed to maintain normal memory abilities. Findings indicate that CBF and its associations with cognition may have utility as a reliable marker of brain function early in the $A D$ process when interventions are likely to be beneficial.

Keywords: aging, Alzheimer's disease, cerebral blood flow, amyloid, arterial spin labeling (ASL), positron emission tomography (PET), neuroimaging, memory

\section{INTRODUCTION}

Cerebral blood flow (CBF) alterations (Bangen et al., 2014) and amyloid- $\beta$ (A $\beta$ ) accumulation (Rodrigue et al., 2012) have been independently linked to increased risk of developing dementia. It is well established that $A \beta$ accumulation is an early event in the Alzheimer's disease (AD) pathological process (Jack et al., 2010, 2013) and there is accumulating evidence of the role of early cerebral vascular dysfunction in AD (Iadecola, 2004; Zlokovic, 2011). This includes disruptions in neurovascular function, which is the normal regulation of CBF by arterioles and the capillary neurovascular unit (Girouard and Iadecola, 2006).

Arterial spin labeling (ASL) is a non-invasive magnetic resonance imaging (MRI) technique in which arterial water is magnetically labeled and used as an endogenous tracer to measure CBF (Detre and Alsop, 1999). ASL has been used to reliably measure $\mathrm{CBF}$ in $\mathrm{AD}$ patients (Johnson et al., 2005); individuals with mild cognitive impairment (MCI) (Bangen et al., 2012); and cognitively normal older adults (Bangen et al., 2009). ASL studies of individuals with AD demonstrate similar patterns of regional hypoperfusion as those shown with studies using fluorodeoxyglucose positron emission tomography (FDG-PET) and single photon emission computed tomography (SPECT) (Chen et al., 2011; Takahashi et al., 2014). ASL techniques have advantages over PET and SPECT including (1) non-invasive use of an endogenous tracer rather than an intravenously administered contrast agent; (2) relatively brief scan times (typically $5-10 \mathrm{~min}$ ) and can be repeated in short succession due to the magnetization of the labeled blood water that decays within seconds; and (3) quantitative measurement of $\mathrm{CBF}$ at rest or during a functional task (Johnson et al., 2005). These advantages along with its increased sensitivity and ability to quantitatively measure perfusion make it ideal to extend its applications for research and in clinical settings (Telischak et al., 2015) designed to monitor neural and vascular changes in healthy aging and disease.

Previous studies have reported associations between $A \beta$ deposition and CBF among older adults across the cognitive spectrum from normal aging to $\mathrm{AD}$. For example, among 182 Alzheimer's Disease Neuroimaging Initiative (ADNI) participants, Mattsson et al. (2014) reported that higher cortical A $\beta$ load measured by florbetapir PET imaging was associated with reduced $\mathrm{CBF}$ in several regions of interest, independent of diagnostic group (cognitively normal, early MCI, late MCI, or AD) (Mattsson et al., 2014). Further, they reported that associations of $A \beta$ load with $C B F$ and brain volume varied across the disease stages. Specifically, in normally aging participants, higher $\mathrm{A} \beta$ load was associated with reduced CBF; however, in individuals with late $\mathrm{MCI}$ and dementia, higher $\mathrm{A} \beta$ load was related to greater reductions of gray matter volume. Given these findings, it was hypothesized that $\mathrm{A} \beta$ pathology may lead to reduced $\mathrm{CBF}$ early in the disease process and volumetric changes later in the disease process, although longitudinal studies are needed to confirm these temporal relationships (Mattsson et al., 2014). In another study including a sample of 27 cognitively normal older adults and 16 individuals diagnosed with amnestic MCI, Michels et al. (2016) reported a trend toward lower global $\mathrm{CBF}$ among those that had greater $\mathrm{A} \beta$ deposition measured with Pittsburgh Compound B (PiB) PET (Michels et al., 2016). Taken together, these studies suggest that CBF may be an important mechanism leading to cognitive decline, and may play an even more prominent role among those with elevated $A \beta$ load.

Findings from several postmortem studies (Arriagada et al., 1992; Ingelsson et al., 2004) and in vivo PET imaging studies (Engler et al., 2006; Jack et al., 2009) have found no significant association between fibrillar amyloid load and degree of cognitive impairment in individuals with $\mathrm{AD}$ dementia. As such, it is thought that fibrillar aggregates of $A \beta$ may not be the immediate cause of cognitive decline and/or $A \beta$ accumulation may be an early event in the $\mathrm{AD}$ pathological cascade and may plateau before onset of dementia (Hampel, 2013). If $A \beta$ accumulation is most dynamic before onset of dementia, its effects on cognition should be studied prior to the onset of significant cognitive decline (Hampel, 2013). Although several postmortem and amyloid PET studies have shown that a considerable portion of asymptomatic older adults have increased $A \beta$ burden in the absence of any cognitive impairment (Price and Morris, 1999; Fagan et al., 2006), other previously published reports have shown statistically significant associations between increased $A \beta$ load on PET and poorer cognitive performance in cognitively normal older adults (Rentz et al., 2011). These effects may be best detected on challenging episodic memory tasks and may interact with various $\mathrm{AD}$ risk factors such as genetic risk (Pike et al., 2011; Kantarci et al., 2012). Little is known about how CBF and A $\beta$, which may both serve as early markers of $\mathrm{AD}$ changes, may interact to affect cognition in cognitively normal older adults.

There is growing evidence supporting the notion that ASL MRI may be a useful biomarker in predicting cognitive decline and progression to MCI and dementia (Chao et al., 2010; BeasonHeld et al., 2013). However, most previous studies have focused on individuals already demonstrating cognitive impairment (MCI and $\mathrm{AD}$ ) and, to our knowledge, no study has considered how ASL MRI CBF and A $\beta$ status may interact to affect cognition 
in cognitively normal older adults. Therefore, we examined potential statistical interactions of ASL MRI CBF and A $\beta$ status on cognitive function within a sample of normally aging older adults drawn from the ADNI study.

\section{MATERIALS AND METHODS}

\section{The ADNI Dataset}

Data used in the preparation of this article were obtained from the ADNI database ${ }^{1}$. The ADNI was launched in 2003 as a public-private partnership, led by Principal Investigator Michael W. Weiner, MD. The primary goal of ADNI has been to test whether serial MRI, PET, other biological markers, and clinical and neuropsychological assessment can be combined to measure the progression of MCI and early AD.

\section{Participants}

Participants were cognitively normal older adults from the ADNI-2 ASL substudy. All participants included in ADNI-2 were between the ages of 55 and 90 years old, had completed at least 6 years of education, were fluent in Spanish or English, and were free of any significant neurological disease other than AD. ADNI control participants had Mini-Mental Status Examination scores $\geq 24$ and Clinical Dementia Rating score of 0 . Full criteria for ADNI eligibility and diagnostic classifications are described in detail at http://www.adni-info.org/Scientists/ ADNIGrant/ProtocolSummary.aspx. This study was approved by the Institutional Review Boards of all of the participating institutions. Informed written consent was obtained from all participants at each site.

Of the 80 control participants who underwent ASL scanning, we included those individuals who had processed data available for download as of September 2016. We further excluded individuals who failed the ADNI raw quality control assessment of ASL data $(n=6)$, were missing PET data $(n=1)$, or were classified as normal controls in ADNI but met criteria for MCI according to comprehensive neuropsychological criteria that operationalizes impairment as performance falling greater than one standard deviation below normative expectations on at least two measures within a cognitive domain $(n=11)$ (Jak et al., 2009; Bondi et al., 2014; Edmonds et al., 2015). This resulted in a final sample of 62 individuals for statistical analyses. The following six measures of cognition were used when diagnosing and excluding for MCI using comprehensive neuropsychological criteria: (1) Animal Fluency, total score; (2) 30-item Boston Naming Test (BNT) total score; (3) Trail Making Test, Part A; time to completion, (4) TMT, Part B; time to completion, (5) Rey Auditory Verbal Learning Test (AVLT) 30-min delayed free recall; number of words recalled, and (6) AVLT recognition; number of words correctly recognized. These measures were selected given their frequent use in assessing early cognitive changes in $\mathrm{AD}$, they were administered to all participants, and they assessed three different domains of cognition - language (Animal Fluency, BNT), speed/executive function (Trail Making

${ }^{1}$ adni.loni.usc.edu
Test, Parts A and B), and episodic memory (AVLT recall and recognition).

\section{Memory Variable Construction}

The AVLT assesses an individual's abilities to acquire 15 words across five immediate learning trials, to recall the words immediately after an intervening interference list (Trial 6), and to recall and recognize the words after a 30-min delay. On verbal serial list-learning tasks, individuals with $\mathrm{AD}$ often show a profile involving rapid forgetting after the introduction of an interference trial and profligate responding to delay recognition foils such that overall performance is often at the level of chance (Libon et al., 2011). As such, in addition to AVLT 30min delayed free recall total number of words recalled and recognition total hits, we calculated additional memory variables to more accurately capture this profile. These additional variables included (1) a post-interference recall score identified as loss of information from Trial 5 to Trial 6 (Mitrushina et al., 1991) and (2) a corrected recognition score considering the number of false positive errors (calculated as [number of recognition hits number of false positives]). Raw neuropsychological scores for each participant were converted into $z$-scores.

\section{Arterial Spin Labeling MRI Data Acquisition and Processing}

Magnetic resonance imaging was performed on a 3.0 Tesla MR scanners from a single vendor (MAGNETOM Trio, Verio, and Skyra, Siemens). A resting state pulsed ASL scan was acquired utilizing QUIPS II with thin-slice TI1 periodic saturation sequence (“Q2TIPS”) with echo-planar imaging (Luh et al., 1999). The sequence included the following parameters: inversion time of arterial spins (TI1) $700 \mathrm{~ms}$, total transit time of the spins (TI2) $1900 \mathrm{~ms}$, tag thickness $100 \mathrm{~mm}$, tag to proximal slice gap $25.4 \mathrm{~mm}$, repetition time $3400 \mathrm{~ms}$, echo time $12 \mathrm{~ms}$, field of view $256 \mathrm{~mm}, 64 \times 64$ matrix, $244 \mathrm{~mm}$ thick axial slices [52 tag + control image pairs], time lag between slices $22.5 \mathrm{~ms}$.

Detailed information describing the ASL MRI data acquisition and processing is available online at www.loni.usc.edu. Briefly, the pipeline involves motion correction, aligning each ASL frame to the first frame using a rigid body transformation, and least squares fitting using SPM8. Perfusion weighted images are computed as the difference between the mean of tagged and untagged ASL data sets. Perfusion weighted images were intensity scaled in order to account for signal decay during acquisition and to allow for intensities in meaningful physiological units. After geometric distortion correction, ASL images were aligned to structural T1-weighted images. Given that we are interested in $\mathrm{CBF}$ in gray matter and therefore want to minimize the effects of the lower perfusion in white matter on our CBF estimates, a partial volume correction was performed that assumes that CBF in gray matter is 2.5 times greater than in white matter. The partial volume corrected perfusion weighted images were normalized by the reference image (i.e., an estimate of blood water magnetization) to convert the signal into physical units (mL/100 g tissue/min). Quality control procedures include inspecting image quality and rating quality as pass or fail. 
FreeSurfer was used to generate anatomical regions of interest (ROIs) for the CBF data and, for secondary analyses, cortical thickness and volume and data for these ROIs. We examined the following four a priori ROIs: (1) hippocampus, (2) posterior cingulate, (3) precuneus, and (4) postcentral gyrus. The first three ROIs were selected because they have been implicated in early $\mathrm{AD}$. These regions are part of the neural network subserving episodic memory function and substantially overlap with the default mode network (Hampel, 2013). It's thought that lifetime cerebral metabolism associated with default activity may predispose these regions to $\mathrm{AD}$-related alterations including $\mathrm{A} \beta$ deposition and disrupted connections with the medial temporal lobe which leads to memory impairment (Buckner et al., 2005). A postcentral ROI was selected to serve as a control region, as we do not expect changes in this region in early AD. Mean CBF corrected for partial volume effects was extracted for each of the four ROIs for each hemisphere separately. Mean CBF for each ROI was calculated by averaging the mean CBF of each hemisphere, with each hemisphere's contribution to the average weighted by the surface area of the ROI for that hemisphere.

\section{Florbetapir PET Data Acquisition and Processing}

A detailed description of ADNI florbetapir PET imaging data acquisition and processing can be found online ${ }^{2}$. Briefly, florbetapir scans were reviewed for quality control before being co-registered, averaged, reoriented into a standard $160 \times 160 \times 96$ voxel image grid with $1.5 \mathrm{~mm}$ cubic voxels, and smoothed to a uniform isotropic resolution of $8 \mathrm{~mm}$ full width at half maximum. Structural MR images were skull-stripped, segmented, parcellated using FreeSurfer and subsequently coregistered to each participant's first florbetapir image.

A florbetapir mean cortical summary standardized uptake value ratio (SUVR) was calculated by averaging across the four main cortical regions (i.e., frontal, anterior/posterior cingulate, lateral parietal, and lateral temporal cortices) and dividing by the mean florbetapir value of the whole cerebellum (white and gray matter). Increased retention of florbetapir is thought to reflect greater cortical $A \beta$ load. $A \beta$ positivity versus negativity was determined using the recommended threshold for cross-sectional florbetapir analyses of 1.11 using the whole cerebellum as the reference region (Clark et al., 2012; Joshi et al., 2012; Landau et al., 2013, 2014). In total, $76 \%$ of the sample $(n=47)$ was determined to be $\mathrm{A} \beta$ negative, while $24 \%$ met criteria for $\mathrm{A} \beta$ positivity $(n=15)$.

\section{Statistical Analyses}

Chi-squared analyses were utilized to compare the groups in terms of categorical variables and analysis of variance (ANOVA) was used for continuous variables. Hierarchical linear regressions were performed to determine the main effects and interaction of $\mathrm{A} \beta$ status (positive or negative) and CBF ROIs on memory performance. For these hierarchical regression analyses, age, education, and sex were the independent variables entered in block 1; CBF of ROIs and $\mathrm{A} \beta$ status were predictors entered in

\footnotetext{
${ }^{2}$ www.loni.usc.edu
}

block 2; and the interaction term was entered in block 3. Memory variables served as the dependent variable in all regression models. Separate regression models were run for each of the four a priori ROIs.

We ran two sets of secondary analyses. First, we ran secondary analyses using the same hierarchical regression models described above but examining $A \beta$ as a continuous variable (i.e., SUVR for the a priori ROIs) rather than as a binary variable (i.e., positive versus negative). Second, we ran additional secondary analyses using the same hierarchical regression models described above but also including APOE genotype ( $\varepsilon 4$ carrier versus noncarrier), pulse pressure (i.e., brachial systolic blood pressure minus diastolic blood pressure), and volume (for hippocampus) or cortical thickness (for posterior cingulate and precuneus) for the a priori ROI in addition to the demographic variables on block 1. APOE genotype and pulse pressure, a measure of arterial stiffening, are two $\mathrm{AD}$ risk factors that are thought to relate to $\mathrm{A} \beta$ accumulation and cerebrovascular functioning (Zlokovic, 2011; Bell et al., 2012; Nation et al., 2015). We also adjusted for volume or cortical thickness of the a priori ROI of the CBF variable in the model to minimize the potential influence of structural brain changes on findings.

For all analyses, the sign of the post-interference recall score and the Trail Making Test variables was reversed during calculation of $z$-scores to be consistent with the other neuropsychological measures (i.e., higher scores reflect better performance). One $A \beta$ negative participant was not administered AVLT Trial 6 and, therefore, this individual was not included in statistical analyses examining the post-interference recall score (i.e., Trial 5 minus Trial 6). All analyses were performed using the Statistical Package for the Social Sciences (SPSS) version 23 (SPSS IBM, Armonk, NY, United States).

\section{RESULTS}

\section{Participant Characteristics}

Participant demographics are presented in Table 1. The A $\beta$ positive group was significantly older, reported fewer years of education, and had a greater proportion of APOE $\varepsilon 4$ carriers in comparison to the $\mathrm{A} \beta$ negative group (all $p$-values $\leq 0.004$ ). There were no significant group differences with respect to sex, pulse pressure, and cognitive performances across the language, executive functioning, and memory measures ( $p$-values $>0.05$ ). There were also no differences between $\mathrm{A} \beta$ positive or negative individuals in terms of CBF in any of the ROIs ( $p$-values $>0.05$ ).

\section{Interaction of Amyloid- $\beta$ and CBF of AD-Vulnerable Regions on Memory Performance}

A series of multiple hierarchical linear regression models adjusting for age, education, and sex were first performed to determine whether there was an interaction between $\mathrm{A} \beta$ status and $\mathrm{CBF}$ of the ROIs on the post-interference recall score (i.e., computed as Trial 5 minus Trial 6). Regression analyses revealed there were significant interactions of $\mathrm{A} \beta$ status and $\mathrm{CBF}$ in 
TABLE 1 | Demographic and neuropsychological characteristics of amyloid negative and amyloid positive groups.

\begin{tabular}{|c|c|c|c|c|c|}
\hline & $\begin{array}{c}\text { Amyloid- } \beta \\
\text { negative }(n=47)\end{array}$ & $\begin{array}{c}\text { Amyloid- } \beta \\
\text { positive }(n=15)\end{array}$ & $F$ or $\mathrm{X}^{2}$ & Significance & Effect size \\
\hline \multicolumn{6}{|l|}{ Demographics } \\
\hline Age, years, mean (SD) & $70.5(5.8)$ & $76.6(6.8)$ & $F=11.6$ & $p=0.001$ & $\eta_{p}^{2}=0.16$ \\
\hline Education, years, mean (SD) & $17.0(2.4)$ & $14.1(3.1)$ & $F=13.7$ & $p<0.001$ & $\eta_{p}^{2}=0.19$ \\
\hline Sex, M:F, (\% female) & $18: 29(61.7 \%)$ & $4: 11(73.3 \%)$ & $x^{2}=0.7$ & $p=0.41$ & $\varphi_{C}=0.10$ \\
\hline APOE $\varepsilon 4,+:-,(\%+)$ & $12: 35(25.5 \%)$ & $10: 5(66.7 \%)$ & $x^{2}=8.4$ & $p=0.004$ & $\varphi_{C}=0.37$ \\
\hline Pulse pressure, mmHg, mean (SD) & $60.5(16.1)$ & $67.5(11.5)$ & $F=2.4$ & $p=0.13$ & $\eta_{p}^{2}=0.04$ \\
\hline \multicolumn{6}{|l|}{ Cognitive measures (z-score)* mean (SD) } \\
\hline \multicolumn{6}{|l|}{ Language } \\
\hline Animal Fluency & $0.11(1.03)$ & $-0.35(0.83)$ & $F=0.05$ & $p=0.83$ & $\eta_{p}^{2}=0.001$ \\
\hline Boston Naming Test & $0.00(1.05)$ & $0.01(0.87)$ & $F=2.13$ & $p=0.15$ & $\eta_{p}^{2}=0.04$ \\
\hline \multicolumn{6}{|l|}{ Attention/Executive function } \\
\hline Trail Making Test, Part A** & $0.18(0.86)$ & $-0.57(1.21)$ & $F=2.47$ & $p=0.12$ & $\eta_{p}^{2}=0.04$ \\
\hline Trail Making Test, Part B** & $0.17(0.91)$ & $-0.52(1.09)$ & $F=0.99$ & $p=0.32$ & $\eta_{p}^{2}=0.02$ \\
\hline \multicolumn{6}{|l|}{ Memory } \\
\hline AVLT Recall Total Correct & $0.11(1.01)$ & $-0.35(0.93)$ & $F=0.45$ & $p=0.51$ & $\eta_{p}^{2}=0.008$ \\
\hline AVLT Post-interference Recall (Trial 5-Trial 6)** & $0.07(0.91)$ & $-0.23(1.25)$ & $F=0.07$ & $p=0.79$ & $\eta_{p}^{2}=0.001$ \\
\hline AVLT Recognition Total Hits & $0.01(0.98)$ & $-0.04(1.10)$ & $F=1.02$ & $p=0.32$ & $\eta_{p}^{2}=0.02$ \\
\hline AVLT Recognition Corrected Total (Hits-False Positives) & $0.05(0.87)$ & $-0.17(1.35)$ & $F=0.68$ & $p=0.41$ & $\eta_{p}^{2}=0.01$ \\
\hline
\end{tabular}

SD, standard deviation; APOE, apolipoprotein E; AVLT, Rey Auditory Verbal Learning Test.

*Results from analysis of covariance (ANCOVAs) comparing the amyloid- $\beta$ positive and negative groups on cognitive measures adjusted for age, education, and gender.

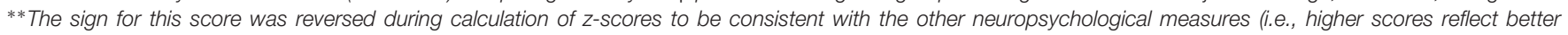
performance).

One A $\beta$ negative participant was not administered AVLT Trial 6 and, therefore, not included in statistical analyses examining the forgetting after interference variable (i.e., Trial 5 minus Trial 6).

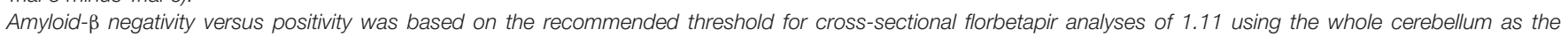
reference region.

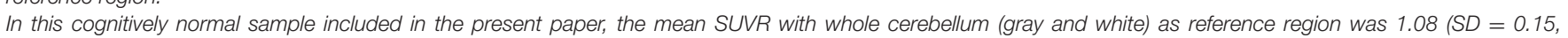

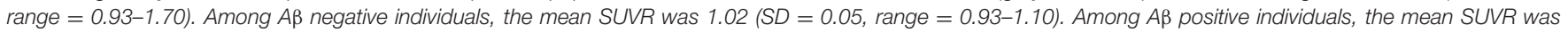

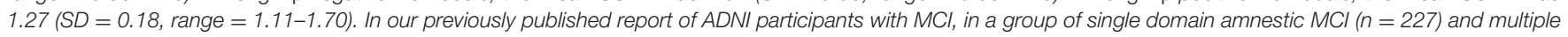

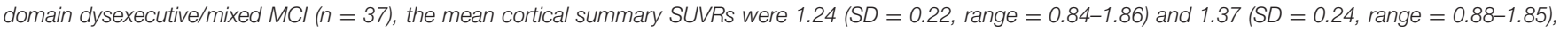

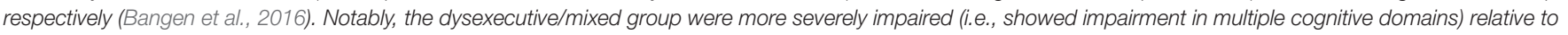

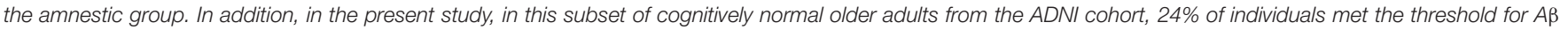

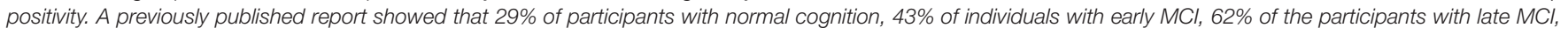
and $77 \%$ of those with Alzheimer's disease were A positive on florbetapir PET imaging in the ADNI cohort (Landau et al., 2012).

the hippocampus $\left[\Delta F(1,54)=8.28, p=0.006, \Delta R^{2}=0.11\right.$, $B=-0.11]$, posterior cingulate $[\Delta F(1,54)=5.04, p=0.03$, $\left.\Delta R^{2}=0.07, B=-0.06\right]$, and precuneus $[\Delta F(1,54)=9.97$, $\left.p=0.003, \Delta R^{2}=0.13, B=-0.08\right]$. Examination of simple main effects using non-parametric tests (Spearman's correlation) revealed there were significant negative associations between post-interference recall memory and CBF of the hippocampus $(\rho=-0.78, p=0.001)$, posterior cingulate $(\rho=-0.64, p=0.01)$, and precuneus $(\rho=-0.65, p=0.009)$ of the $A \beta$ positive group; however, there were no significant associations between post-interference recall memory and $\mathrm{CBF}$ of the hippocampus ( $\rho=-0.14, p=0.37)$, posterior cingulate $(\rho=-0.04, p=0.81)$, and precuneus $(\rho=-0.17, p=0.25)$ in the $\mathrm{A} \beta$ negative group (See Figure $\mathbf{1}$ and Table 2 ). When secondary analyses were performed with $\mathrm{A} \beta$ as a continuous variable (i.e., SUVR for the a priori ROI) rather than as a binary variable (i.e., positive versus negative) regression analyses revealed there was a significant interaction of $A \beta$ and $C B F$ of the precuneus $\left[\Delta F(1,54)=6.97, p=0.01, \Delta R^{2}=0.10, B=-0.18\right]$. Interactions of $\mathrm{A} \beta$ and $\mathrm{CBF}$ in the hippocampus and posterior cingulate were attenuated and no longer statistically significant $\left[\Delta F(1,54)=3.61, p=0.06, \Delta R^{2}=0.05, B=-0.29\right]$ and posterior cingulate $\left[\Delta F(1,54)=1.49, p=0.23, \Delta R^{2}=0.02\right.$, $B=-0.08]$.

When additional secondary analyses adjusting for APOE genotype ( $\varepsilon 4$ carrier versus non-carrier), pulse pressure, and volume or cortical thickness of the a priori ROI were performed, results remained qualitatively and statistically similar to the findings for the primary analyses reported above. There were no main effects of $\mathrm{A} \beta$ status or $\mathrm{CBF}$ on post-interference recall memory (all $p$-values $>0.05$ ) for any ROI. For delayed recall memory as assessed as total number of words correctly recalled after a 30-min delay, there were no main effects or interactions (all $p$-values $>0.05$ ).

A second set of multiple linear regressions were performed to determine whether there was an interaction between $A \beta$ status and $\mathrm{CBF}$ of our ROIs for recognition memory performance (total hits minus false positive errors). Regression analyses adjusting for age, education, and sex, revealed there were significant interactions of $\mathrm{A} \beta$ status and $\mathrm{CBF}$ in the hippocampus 


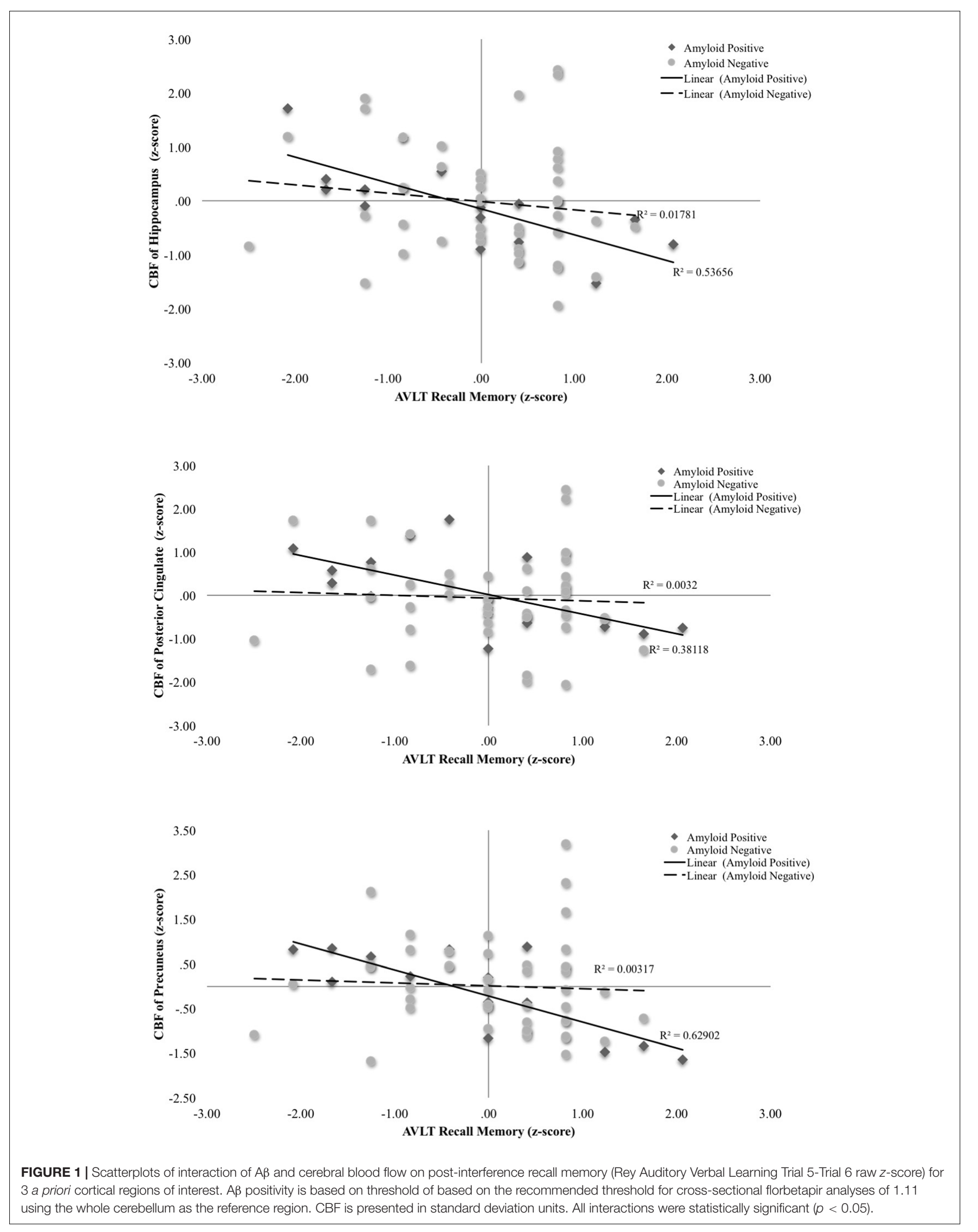




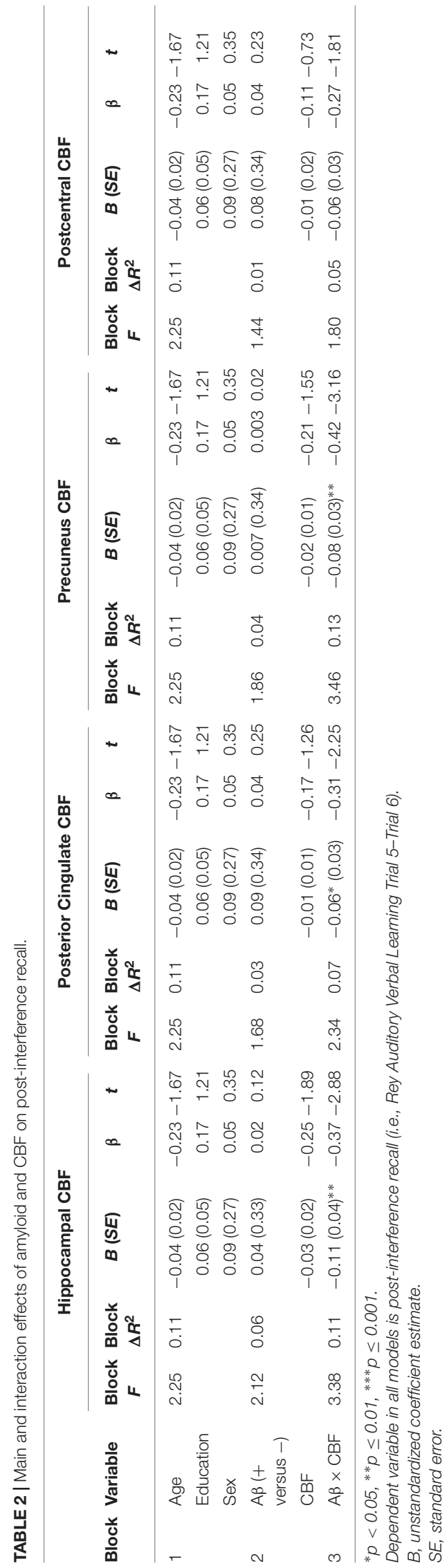

$\left[\Delta F(1,55)=11.98, p=0.001, \Delta R^{2}=0.15, B=-0.13\right]$, posterior cingulate $\left[\Delta F(1,55)=7.92, p=0.007, \Delta R^{2}=0.11, B=-0.07\right]$, and precuneus $\left[\Delta F(1,55)=6.35, p=0.015, \Delta R^{2}=0.09\right.$, $B=-0.07]$. Examination of simple main effects using nonparametric tests (Spearman's correlation) revealed there were significant negative associations between recognition memory performance and $\mathrm{CBF}$ of the hippocampus $(\rho=-0.57, p=0.03)$ and posterior cingulate $(\rho=-0.59, p=0.02)$ in the $A \beta$ positive group. There was a trend toward worse recognition memory performance and higher $\mathrm{CBF}$ of the precuneus in the $\mathrm{A} \beta$ positive group $(\rho=-0.46, p=0.09)$. There were no significant associations between recognition memory and CBF of the hippocampus ( $\rho=0.13, p=0.39)$, posterior cingulate $(\rho=0.25$, $p=0.09)$, or precuneus $(\rho=0.17, p=0.25)$ in the $\mathrm{A} \beta$ negative group (see Figure 2 and Table 3). When secondary analyses were performed with $A \beta$ as a continuous variable (i.e., SUVR for the a priori ROIs) rather than as a binary variable (i.e., positive versus negative), results remained similar. Regression analyses revealed there were significant interactions of $\mathrm{A} \beta$ and $\mathrm{CBF}$ in the hippocampus $\left[\Delta F(1,55)=18.62, p<0.001, \Delta R^{2}=0.21\right.$, $B=-0.58]$, posterior cingulate $[\Delta F(1,55)=12.69, p=0.001$, $\left.\Delta R^{2}=0.16, B=-0.22\right]$, and precuneus $[\Delta F(1,55)=21.13$, $\left.p<0.001, \Delta R^{2}=0.24, B=-0.28\right]$.

When additional secondary analyses adjusting for APOE genotype ( $\varepsilon 4$ carrier versus non-carrier), pulse pressure, and volume or cortical thickness of the a priori ROIs were performed, results remained qualitatively and statistically similar to the findings for the primary analyses reported above. There were no main effects of $A \beta$ status or CBF on post-interference recall memory (all $p$-values $>0.05$ ) for any ROI. For delayed recall memory as assessed as total number of words correctly recalled after a 30-min delay, there were no main effects or interactions (all $p$-values $>0.05$ ). In addition, findings were qualitatively and statistically similar when total recognition hits (i.e., not considering false positives) served as the dependent variable. As hypothesized, there were no interactions of $A \beta$ status and postcentral CBF on memory performance (all $p$-values $>0.05$ ). In addition, there were no main effects of $\mathrm{A} \beta$ status or $\mathrm{CBF}$ on recognition memory performance (all $p$-values $>0.05$ ) for any ROI.

\section{DISCUSSION}

Our study extends previous CBF studies of dementia risk by showing statistical interactions between $A \beta$ status (negative or positive) and regional $\mathrm{CBF}$ on memory performance in a sample of well-characterized, cognitively normal older adults. Specifically, we found that among $A \beta$ positive older adults, there were significant associations between higher $\mathrm{CBF}$ and poorer verbal memory performance in regions known to be predilections sites for $\mathrm{AD}$-the hippocampus, posterior cingulate, and precuneus. In contrast, among $A \beta$ negative older adults, there were no significant relationships between memory performance and $\mathrm{CBF}$, although there was a trend toward higher $\mathrm{CBF}$ in the posterior cingulate and better verbal memory performance. Importantly, our findings demonstrate differential 


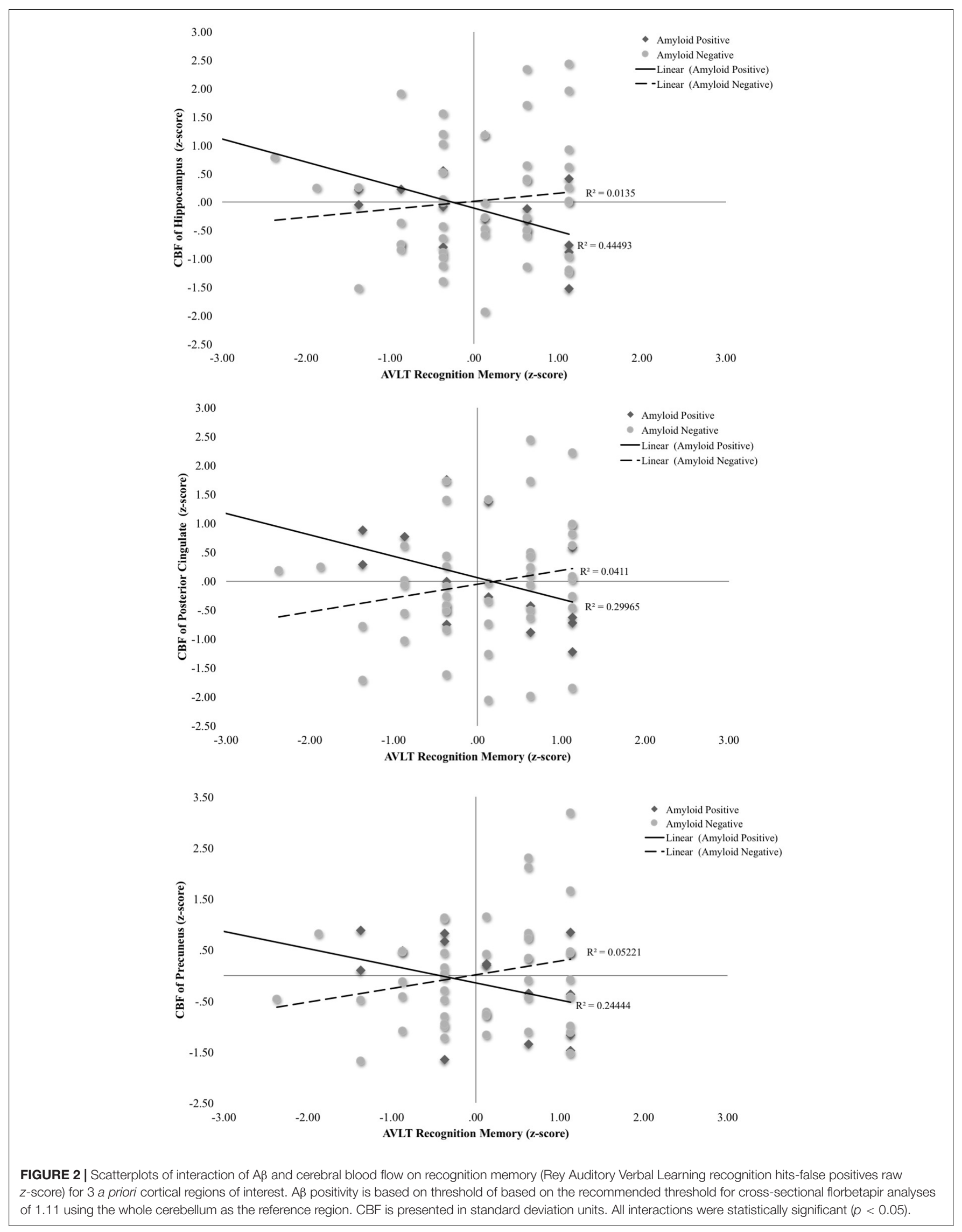




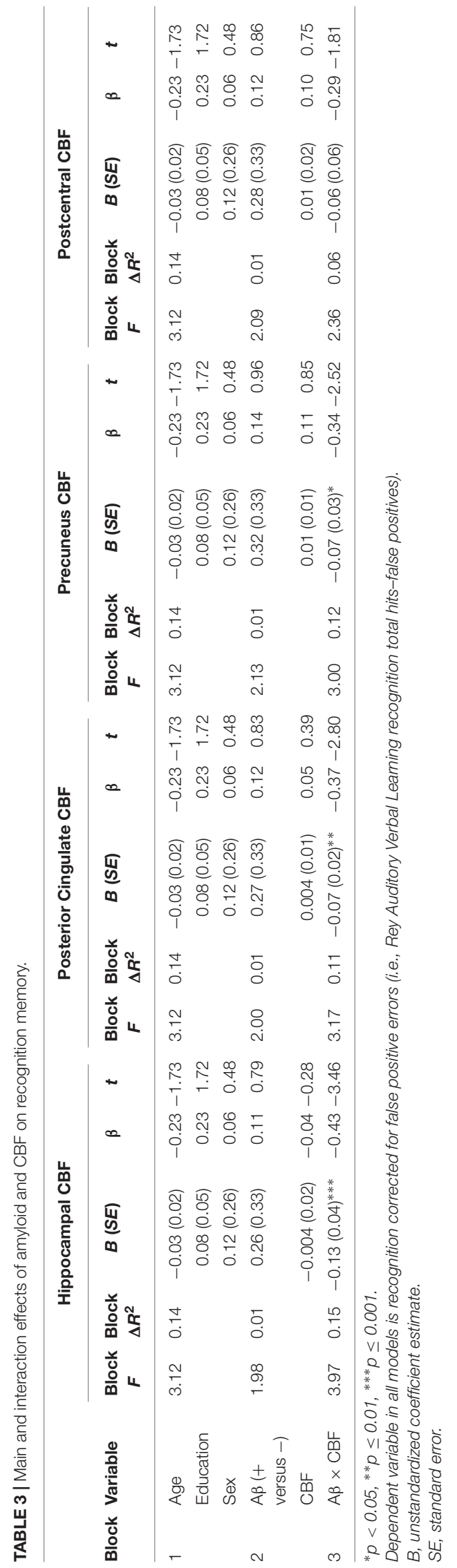

associations between $\mathrm{CBF}$ and cognition for $\mathrm{A} \beta$ positive versus negative cognitively normal older adults.

Although regional decreases in $\mathrm{CBF}$ are interpreted as reflecting decreased brain function, increases in perfusion in the context of preclinical $\mathrm{AD}$-particularly when cognitive performance is maintained or even improved-has often been considered to represent a compensatory response to an incipient pathologic process (Dai et al., 2009). Indeed, several previously published studies have found significant differences in resting hyperperfusion in tandem with better memory function in nondemented older adults at risk for $\mathrm{AD}$, and researchers have interpreted this finding as a potential compensatory response reflecting metabolic alterations and/or increased need for glucose and oxygen to support neuronal activity (Fleisher et al., 2009; Bangen et al., 2012; Zlatar et al., 2014). In contrast, we found that higher resting $\mathrm{CBF}$ was associated with poorer memory performance among older adults at increased risk for $\mathrm{AD}$ by virtue of elevated $A \beta$ accumulation, possibly reflecting cerebrovascular dysregulation or a cellular and/or vascular compensatory response to pathologic processes whereby higher $\mathrm{CBF}$ is needed to maintain normal memory abilities. Unlike our previously published work, all individuals in this study were cognitively normal and, importantly, there were no group differences among the $A \beta$ positive and $A \beta$ negative group in terms of cognitive performance. The heightened CBF in $A \beta$ positive individuals may suggest that these individuals are on a declining trajectory of RAVLT performance (albeit still normal), and they need more CBF to support this declining memory system. Hyperperfusion in early MCI followed by hypoperfusion later in MCI when approaching the transition to dementia has been shown and it is possible that the $A \beta$ positive individuals in our sample are closer to developing MCI. Further longitudinal studies investigating perfusion differences across the course of the disease are needed to further examine the role of higher CBF.

Our work showing statistical interactions of perfusion and $A \beta$ status is consistent with previous studies that have demonstrated links between $A \beta$ and cerebrovascular dysregulation. Specifically, prior work has shown that $A \beta$ increases the vulnerability of the brain to cerebral ischemia through its effects on the cells of the neurovascular unit (Zhang et al., 1997; Iadecola, 2004; Girouard and Iadecola, 2006). Moreover, cerebrovascular dysfunction upregulates amyloid precursor protein and $A \beta$ cleavage (Abe et al., 1991; Yokota et al., 1996; Iadecola, 2004). Ultimately, A $\beta$ and cerebrovascular dysfunction are thought to reinforce one another thereby amplifying their deleterious effects on the brain (Iadecola, 2004). The present findings provide further support for the role of vascular alterations in the $\mathrm{AD}$ prodrome.

Previous studies of cerebral perfusion across the continuum from the preclinical phase to $\mathrm{AD}$ suggest a biphasic pattern characterized by early hyperperfusion preceding later hypoperfusion (Wierenga et al., 2014). In this way, cerebrovascular dysregulation becomes more pronounced over time as the disease progresses (Mentis et al., 1998). This may be due to several factors including neuronal death and synaptic loss resulting in a reduced hemodynamic response to neural activation; accumulating amyloid in cerebral arterioles leading to disruptions in the ability of vascular smooth muscles cells to relax 
thereby creating a mechanical obstacle to vasodilation (Christie et al., 2001); and atherosclerosis in the circle of Willis (Roher et al., 2003) and conduit cerebral arteries resulting in reduced global CBF and further disruption in the ability of neural stimuli to increase perfusion (Iadecola, 2004). Furthermore, evidence suggests that increased activation within neural networks may modulate $A \beta$ accumulation given that brain regions with lifelong high activity levels (e.g., default mode network) also have the greatest predisposition for $A \beta$ accumulation and increased synaptic transmission results in increased interstitial fluid $A \beta$ levels (Cirrito et al., 2008; Hampel, 2013).

Accumulating evidence suggests that ASL CBF represents a useful biomarker in at-risk individuals since this technique can sensitively differentiate those at risk from control participants (Fleisher et al., 2009; Bangen et al., 2012; Wierenga et al., 2012). Additionally, ASL CBF indices have reliably predicted progression from normal cognition to MCI (Beason-Held et al., 2013), and MCI to AD (Chao et al., 2010). Longitudinal studies have shown that, relative to individuals who remained cognitively normal, older adults who later developed MCI demonstrated hyperperfusion in orbitofrontal, medial frontal, and anterior cingulate regions over time, accompanied by reduced $\mathrm{CBF}$ in parietal, temporal, and thalamic regions (Beason-Held et al., 2013). These changes occurred several years prior to the development of cognitive impairment and were observed in regions known to be predilection sites for early $\mathrm{AD}$ pathology (Beason-Held et al., 2013). Additionally, these changes were independent of longitudinal changes in tissue volume. This is consistent with findings from our secondary analyses that revealed significant interactions of $\mathrm{A} \beta$ status and $\mathrm{CBF}$ on memory performance independent of volume or cortical thickness, further suggesting that CBF may play a role in cognitive functioning independent of tissue loss.

In the few existing longitudinal prospective studies using ASL MRI, resting hypoperfusion of the right inferior parietal cortex and right middle frontal cortex at baseline predicted progression from MCI to dementia at 3-year follow-up (Chao et al., 2010) and in another study reduced CBF in the posterior cingulate at baseline was associated with development of cognitive decline at 18-month follow up in healthy older adults (Xekardaki et al., 2015). Our present findings highlight the important association between $\mathrm{CBF}$ and memory, and they provide further support for the notion that $\mathrm{CBF}$ is a useful marker of $\mathrm{AD}$ risk and correlate of cognitive function in older adults. Specifically, we observed evidence of dysregulated $\mathrm{CBF}$ patterns in $\mathrm{A} \beta$ positive individuals who are cognitively normal suggesting that ASL MRI is sensitive to very early changes in the brain.

The present findings suggest that $\mathrm{A} \beta$ accumulation and $\mathrm{CBF}$ alterations together influence memory performance in at-risk older adults. These findings add to a growing body of evidence underscoring the importance of multiple pathological processes co-occurring in $\mathrm{AD}$ and the interactive influence of several risk factors. Neuropathological studies have shown that clinically diagnosed MCI and AD are both pathologically heterogeneous disorders (Schneider et al., 2007; Nettiksimmons et al., 2014). In our own sample of autopsy-confirmed AD, we found that the presence of mild cerebrovascular changes was associated with less severe $\mathrm{AD}$ pathology yet there were no differences in severity of cognitive impairment between the $\mathrm{AD}$ patients with and without evidence of cerebrovascular disease (Bangen et al., 2015). These results raise the possibility that cerebrovascular changes contribute to overall severity of cognitive impairment, even in patients with both autopsy-confirmed $\mathrm{AD}$ and relatively mild cerebrovascular disease (Bangen et al., 2015). We have also shown that the presence of multiple $\mathrm{AD}$ risk factors (e.g., advanced age, APOE $\varepsilon 4$ allele, family history of $\mathrm{AD}$, and/or increased vascular risk burden in different combinations) has additive or interactive effects on brain function and cognition (Fleisher et al., 2009; Bangen et al., 2014). The present findings extend this work by demonstrating interactions between PET brain $\mathrm{A} \beta$ positivity and CBF on memory performance.

Our results did not reveal any significant main effects of $\mathrm{A} \beta$ status or $\mathrm{CBF}$ on memory performance in our sample. With respect to $A \beta$, findings from cross-sectional studies have been inconsistent with some studies reporting no relationship between burden of amyloid in the brain and cognition in cognitively normal or non-demented older adults (Mintun et al., 2006; Aizenstein et al., 2008; Mormino et al., 2009; Rowe et al., 2010) whereas other studies showed associations between greater amyloid and worse cognition (Rodrigue et al., 2012). Additionally, other studies have showed relationships between greater amyloid and worse cognition in APOE $\varepsilon 4$ carriers, while no such relationship (Lim et al., 2013) or a weaker relationship among non-carriers (Kantarci et al., 2012).

Prospective longitudinal studies have also been mixed with some studies reporting greater faster rates of cognitive decline in non-demented older adults with high cerebral $A \beta$ load over an 18-month period following PET imaging (Doraiswamy et al., 2012; Lim et al., 2012; Ellis et al., 2013; Kawas et al., 2013) whereas other studies have found no difference in rate of cognitive change over 2- to 3-year follow-up between cognitively normal older adults who had high versus low $\mathrm{A} \beta$ at baseline (Villemagne et al., 2011; Ewers et al., 2012). However, prospective longitudinal studies have generally had little follow up after PET imaging (Gu et al., 2015), and retrospective longitudinal studies have shown that non-demented older adults who have higher levels of $\mathrm{A} \beta$ showed faster cognitive decline prior to PET scanning relative to their counterparts with lower level of $A \beta$ (Resnick et al., 2010; Landau et al., 2012; Gu et al., 2015). A metaanalysis of 64 studies examining amyloid-cognition associations in healthy older adults found that episodic memory had a small and significant relationship to amyloid burden whereas other cognitive abilities (e.g., working memory, processing speed, visuospatial function, semantic memory) did not have significant relationships to amyloid. Study design, that is cross-sectional vs. longitudinal design, had little influence on findings (Hedden et al., 2013). Although the role of $A \beta$ in cognitive decline and the clinical expression of $\mathrm{AD}$ is complex and may be moderated by additional risk factors and variables (Kantarci et al., 2012; Lim et al., 2013; Gu et al., 2015), there is clear evidence to suggest it contributes to the $\mathrm{AD}$ process and pivotal to the amyloid cascade model (Jack et al., 2010, 2013).

In contrast to our current ADNI-based findings, we have previously found in our own community samples main effects 
of CBF on cognition when examining both cognitively normal older adults and those with MCI (Bangen et al., 2012, 2014). In the present sample of ADNI participants, all individuals were cognitively normal and, given selection criteria for ADNI, all participants had very low vascular risk burden. It is possible that we would have found main effects of CBF on memory if there were a greater range of cognitive performance and $\mathrm{CBF}$ values. A previously published paper in the ADNI cohort found that the effects of higher brain $A \beta$ load was associated with reduced $\mathrm{CBF}$ in cognitively normal older adults and with reduced brain volume in late $\mathrm{MCI}$ and dementia suggesting that the relationship between $A \beta$ and $C B F$ changes over the course of the disease (Mattsson et al., 2014). In the current study, we focused on the interaction between $A \beta$ and CBF on memory and it is possible that we would have observed different relationships among $\mathrm{A} \beta$ status, $\mathrm{CBF}$, and memory performance if we included participants with more pronounced cerebrovascular disease and/or individuals with MCI or AD. However, given a critical need to examine preclinical AD in its very earliest stages, for the purposes of the current study we emphasized associations among $\mathrm{A} \beta$ status, $\mathrm{CBF}$ and memory function in older adults who show brain $A \beta$ positivity on PET in the context of no detectable cognitive impairment.

This work has several important research and clinical implications. First, our findings suggest a dynamic relationship between cerebral perfusion and $A \beta$ in the expression of memory function in individuals with preclinical AD. Results further underscore the potential value in examining sensitive vascular variables in the pathogenesis of $\mathrm{AD}$. Additionally, pharmacological and behavioral interventions, including physical exercise, may play a critical role in the regulation of $\mathrm{CBF}$ and, ultimately, the prevention of cognitive decline. Interestingly, a recent study showed that older adults taking angiotensin II AT1-receptor blockers exhibited reduced cerebral amyloid retention (Nation et al., 2016). As noted by the authors, this finding is consistent with results from studies in transgenic animals, and they may explain in part why older adults who use AT1-receptor blockers show reduced progression to dementia despite greater vascular risk burden (Nation et al., 2016). Future research is needed to further determine whether antihypertension medication and/or behavioral lifestyle changes may improve cerebral microcirculation and reduce $A \beta$ retention.

Strengths of this study include a well-characterized sample of older adults who have undergone multi-modal neuroimaging and neuropsychological assessment as part of a national study on aging and AD. Limitations of our study include use of a global measurement of $A \beta$ pathology rather than local or regional measures. In addition, this was a cross-sectional study and we did not assess cognitive outcome. It is possible that some of the $\mathrm{A} \beta$ positive individuals in this study will not develop $\mathrm{AD}$ and, likewise, some of the $A \beta$ negative individuals may express the disease at some point. Furthermore, previously published results have reported an absence of cross-sectional associations between amyloid and cognition in healthy controls but have found negative associations for when data is examined longitudinally (Gu et al., 2015). Despite these limitations, in the search for reliable biomarkers of very early $\mathrm{AD}$, ASL MRI may prove especially useful, and the combination of both cerebrovascular and $\mathrm{A} \beta$ markers may more completely inform the complex pathological processes underlying the clinical expression of AD than either biomarker class alone. Finally, since vascular risk factors are modifiable, these results may have important implications for biomarker studies, clinical trials, and treatment.

\section{AUTHOR CONTRIBUTIONS}

KB designed the study, analyzed and interpreted the data, and wrote and revised the manuscript. AC analyzed and interpreted the data and wrote and revised the manuscript. EE, NE, MW, KT, LL, MT, ZZ, DN, MB, and LD-W interpreted the data and revised the manuscript for important intellectual contact. All authors approved the submitted version of the manuscript and agree to be accountable for all aspects of the work.

\section{FUNDING}

This work was supported by VA Clinical Science Research and Development (Career Development Award-2 1IK2CX000938 to $\mathrm{KB}$ and 1IK2CX001415 to EE), the Alzheimer's Association (NIRG-15-364251 to KB), and NIH (K24 AG026431 to MB; R01 AG049810 to MB, EE, and LD-W; and K23AG049906 to ZZ).

\section{ACKNOWLEDGMENTS}

Data collection and sharing for this project was funded by the ADNI (National Institutes of Health Grant U01 AG024904) and DOD ADNI (Department of Defense award number W81XWH12-2-0012). ADNI is funded by the National Institute on Aging, the National Institute of Biomedical Imaging and Bioengineering, and through generous contributions from the following: AbbVie, Alzheimer's Association; Alzheimer's Drug Discovery Foundation; Araclon Biotech; BioClinica, Inc.; Biogen; BristolMyers Squibb Company; CereSpir, Inc.; Cogstate; Eisai Inc.; Elan Pharmaceuticals, Inc.; Eli Lilly and Company; EuroImmun; F. Hoffmann-La Roche Ltd. and its affiliated company Genentech, Inc.; Fujirebio; GE Healthcare; IXICO Ltd.; Janssen Alzheimer Immunotherapy Research \& Development, LLC.; Johnson \& Johnson Pharmaceutical Research \& Development LLC.; Lumosity; Lundbeck; Merck \& Co., Inc.; Meso Scale Diagnostics, LLC.; NeuroRx Research; Neurotrack Technologies; Novartis Pharmaceuticals Corporation; Pfizer Inc.; Piramal Imaging; Servier; Takeda Pharmaceutical Company; and Transition Therapeutics. The Canadian Institutes of Health Research is providing funds to support ADNI clinical sites in Canada. Private sector contributions are facilitated by the Foundation for the National Institutes of Health (www.fnih.org). The grantee organization is the Northern California Institute for Research and Education, and the study is coordinated by the Alzheimer's Therapeutic Research Institute at the University of Southern California. ADNI data are disseminated by the Laboratory for Neuro Imaging at the University of Southern California. 


\section{REFERENCES}

Abe, K., Tanzi, R. E., and Kogure, K. (1991). Selective induction of Kunitz-type protease inhibitor domain-containing amyloid precursor protein mRNA after persistent focal ischemia in rat cerebral cortex. Neurosci. Lett. 125, 172-174. doi: 10.1016/0304-3940(91)90020-T

Aizenstein, H. J., Nebes, R. D., Saxton, J. A., Price, J. C., Mathis, C. A., Tsopelas, N. D., et al. (2008). Frequent amyloid deposition without significant cognitive impairment among the elderly. Arch. Neurol. 65, 1509-1517. doi: 10.1001/ archneur.65.11.1509

Arriagada, P. V., Growdon, J. H., Hedley-Whyte, E. T., and Hyman, B. T. (1992). Neurofibrillary tangles but not senile plaques parallel duration and severity of Alzheimer's disease. Neurology 42(3 Pt 1), 631-639. doi: 10.1212/WNL. 42.3.631

Bangen, K. J., Clark, A. L., Werhane, M., Edmonds, E. C., Nation, D. A., Evangelista, N., et al. (2016). Cortical amyloid burden differences across empirically-derived mild cognitive impairment subtypes and interaction with APOE varepsilon4 genotype. J. Alzheimers. Dis. 52, 849-861. doi: 10.3233/jad150900

Bangen, K. J., Nation, D. A., Clark, L. R., Harmell, A. L., Wierenga, C. E., Dev, S. I., et al. (2014). Interactive effects of vascular risk burden and advanced age on cerebral blood flow. Front. Aging Neurosci. 6:159. doi: 10.3389/fnagi.2014. 00159

Bangen, K. J., Nation, D. A., Delano-Wood, L., Weissberger, G. H., Hansen, L. A., Galasko, D. R., et al. (2015). Aggregate effects of vascular risk factors on cerebrovascular changes in autopsy-confirmed Alzheimer's disease. Alzheimers Dement. 11, 394.e-403.e. doi: 10.1016/j.jalz.2013.12.025

Bangen, K. J., Restom, K., Liu, T. T., Jak, A. J., Wierenga, C. E., Salmon, D. P., et al. (2009). Differential age effects on cerebral blood flow and BOLD response to encoding: associations with cognition and stroke risk. Neurobiol. Aging 30, 1276-1287. doi: 10.1016/j.neurobiolaging.2007.11.012

Bangen, K. J., Restom, K., Liu, T. T., Wierenga, C. E., Jak, A. J., Salmon, D. P., et al. (2012). Assessment of Alzheimer's disease risk with functional magnetic resonance imaging: an arterial spin labeling study. J. Alzheimers Dis. 31(Suppl. 3), S59-S74. doi: 10.3233/jad-2012-120292

Beason-Held, L. L., Goh, J. O., An, Y., Kraut, M. A., O’Brien, R. J., Ferrucci, L., et al. (2013). Changes in brain function occur years before the onset of cognitive impairment. J. Neurosci. 33, 18008-18014. doi: 10.1523/jneurosci.140213.2013

Bell, R. D., Winkler, E. A., Singh, I., Sagare, A. P., Deane, R., Wu, Z., et al. (2012). Apolipoprotein E controls cerebrovascular integrity via cyclophilin A. Nature 485, 512-516. doi: 10.1038/nature 11087

Bondi, M. W., Edmonds, E. C., Jak, A. J., Clark, L. R., Delano-Wood, L., McDonald, C. R., et al. (2014). Neuropsychological criteria for mild cognitive impairment improves diagnostic precision, biomarker associations, and progression rates. J. Alzheimers. Dis. 42, 275-289. doi: 10.3233/jad- 140276

Buckner, R. L., Snyder, A. Z., Shannon, B. J., LaRossa, G., Sachs, R., Fotenos, A. F., et al. (2005). Molecular, structural, and functional characterization of Alzheimer's disease: evidence for a relationship between default activity, amyloid, and memory. J. Neurosci. 25, 7709-7717. doi: 10.1523/jneurosci.217705.2005

Chao, L. L., Buckley, S. T., Kornak, J., Schuff, N., Madison, C., Yaffe, K., et al. (2010). ASL perfusion MRI predicts cognitive decline and conversion from MCI to dementia. Alzheimer Dis. Assoc. Disord. 24, 19-27. doi: 10.1097/WAD. 0b013e3181b4f736

Chen, Y., Wolk, D. A., Reddin, J. S., Korczykowski, M., Martinez, P. M., Musiek, E. S., et al. (2011). Voxel-level comparison of arterial spin-labeled perfusion MRI and FDG-PET in Alzheimer disease. Neurology 77, 1977-1985. doi: 10. 1212/WNL.0b013e31823a0ef7

Christie, R., Yamada, M., Moskowitz, M., and Hyman, B. (2001). Structural and functional disruption of vascular smooth muscle cells in a transgenic mouse model of amyloid angiopathy. Am. J. Pathol. 158, 1065-1071. doi: 10.1016/ s0002-9440(10)64053-9

Cirrito, J. R., Kang, J. E., Lee, J., Stewart, F. R., Verges, D. K., Silverio, L. M., et al. (2008). Endocytosis is required for synaptic activity-dependent release of amyloid-beta in vivo. Neuron 58, 42-51. doi: 10.1016/j.neuron.2008.02.003

Clark, C. M., Pontecorvo, M. J., Beach, T. G., Bedell, B. J., Coleman, R. E., Doraiswamy, P. M., et al. (2012). Cerebral PET with florbetapir compared with neuropathology at autopsy for detection of neuritic amyloid-beta plaques: a prospective cohort study. Lancet Neurol. 11, 669-678. doi: 10.1016/s14744422(12)70142-4

Dai, W., Lopez, O. L., Carmichael, O. T., Becker, J. T., Kuller, L. H., and Gach, H. M. (2009). Mild cognitive impairment and alzheimer disease: patterns of altered cerebral blood flow at MR imaging. Radiology 250, 856-866. doi: 10. 1148/radiol.2503080751

Detre, J. A., and Alsop, D. C. (1999). Perfusion magnetic resonance imaging with continuous arterial spin labeling: methods and clinical applications in the central nervous system. Eur. J. Radiol. 30, 115-124. doi: 10.1016/S0720048X(99)00050-9

Doraiswamy, P. M., Sperling, R. A., Coleman, R. E., Johnson, K. A., Reiman, E. M., Davis, M. D., et al. (2012). Amyloid-beta assessed by florbetapir F 18 PET and 18-month cognitive decline: a multicenter study. Neurology 79, 1636-1644. doi: 10.1212/WNL.0b013e3182661f74

Edmonds, E. C., Delano-Wood, L., Clark, L. R., Jak, A. J., Nation, D. A., McDonald, C. R., et al. (2015). Susceptibility of the conventional criteria for mild cognitive impairment to false-positive diagnostic errors. Alzheimers Dement. 11, 415-424. doi: 10.1016/j.jalz.2014.03.005

Ellis, K. A., Lim, Y. Y., Harrington, K., Ames, D., Bush, A. I., Darby, D., et al. (2013). Decline in cognitive function over 18 months in healthy older adults with high amyloid-beta. J. Alzheimers Dis. 34, 861-871. doi: 10.3233/jad-122170

Engler, H., Forsberg, A., Almkvist, O., Blomquist, G., Larsson, E., Savitcheva, I., et al. (2006). Two-year follow-up of amyloid deposition in patients with Alzheimer's disease. Brain 129(Pt 11), 2856-2866. doi: 10.1093/brain/awl178

Ewers, M., Insel, P., Jagust, W. J., Shaw, L., Trojanowski, J. Q., Aisen, P., et al. (2012). CSF biomarker and PIB-PET-derived beta-amyloid signature predicts metabolic, gray matter, and cognitive changes in nondemented subjects. Cereb. Cortex 22, 1993-2004. doi: 10.1093/cercor/bhr271

Fagan, A. M., Mintun, M. A., Mach, R. H., Lee, S. Y., Dence, C. S., Shah, A. R., et al. (2006). Inverse relation between in vivo amyloid imaging load and cerebrospinal fluid Abeta42 in humans. Ann. Neurol. 59, 512-519. doi: 10.1002/ ana. 20730

Fleisher, A. S., Podraza, K. M., Bangen, K. J., Taylor, C., Sherzai, A., Sidhar, K., et al. (2009). Cerebral perfusion and oxygenation differences in Alzheimer's disease risk. Neurobiol. Aging 30, 1737-1748. doi: 10.1016/j.neurobiolaging.2008.01.012

Girouard, H., and Iadecola, C. (2006). Neurovascular coupling in the normal brain and in hypertension, stroke, and Alzheimer disease. J. Appl. Physiol. (1985) 100, 328-335. doi: 10.1152/japplphysiol.00966.2005

Gu, Y., Razlighi, Q. R., Zahodne, L. B., Janicki, S. C., Ichise, M., Manly, J. J., et al. (2015). Brain amyloid deposition and longitudinal cognitive decline in nondemented older subjects: results from a multi-ethnic population. PLoS ONE 10:e0123743. doi: 10.1371/journal.pone.0123743

Hampel, H. (2013). Amyloid-beta and cognition in aging and Alzheimer's disease: molecular and neurophysiological mechanisms. J. Alzheimers Dis. 33(Suppl. 1), S79-S86. doi: 10.3233/jad-2012-129003

Hedden, T., Oh, H., Younger, A. P., and Patel, T. A. (2013). Meta-analysis of amyloid-cognition relations in cognitively normal older adults. Neurology 80, 1341-1348. doi: 10.1212/WNL.0b013e31828ab35d

Iadecola, C. (2004). Neurovascular regulation in the normal brain and in Alzheimer's disease. Nat. Rev. Neurosci. 5, 347-360. doi: 10.1038/nrn1387

Ingelsson, M., Fukumoto, H., Newell, K. L., Growdon, J. H., Hedley-Whyte, E. T., Frosch, M. P., et al. (2004). Early Abeta accumulation and progressive synaptic loss, gliosis, and tangle formation in AD brain. Neurology 62, 925-931. doi: 10.1212/01.WNL.0000115115.98960.37

Jack, C.R. Jr., Knopman, D. S., Jagust, W. J., Petersen, R. C., Weiner, M. W., Aisen, P. S., et al. (2013). Tracking pathophysiological processes in Alzheimer's disease: an updated hypothetical model of dynamic biomarkers. Lancet Neurol. 12, 207-216. doi: 10.1016/s1474-4422(12)70291-0

Jack, C.R. Jr., Knopman, D. S., Jagust, W. J., Shaw, L. M., Aisen, P. S., Weiner, M. W., et al. (2010). Hypothetical model of dynamic biomarkers of the Alzheimer's pathological cascade. Lancet Neurol. 9, 119-128. doi: 10.1016/ s1474-4422(09)70299-6

Jack, C.R. Jr., Lowe, V. J., Weigand, S. D., Wiste, H. J., Senjem, M. L., Knopman, D. S., et al. (2009). Serial PIB and MRI in normal, mild cognitive impairment and Alzheimer's disease: implications for sequence of pathological events in Alzheimer's disease. Brain 132(Pt 5), 1355-1365. doi: 10.1093/brain/awp062 
Jak, A. J., Bondi, M. W., Delano-Wood, L., Wierenga, C., Corey-Bloom, J., Salmon, D. P., et al. (2009). Quantification of five neuropsychological approaches to defining mild cognitive impairment. Am. J. Geriatr. Psychiatry 17, 368-375. doi: 10.1097/JGP.0b013e31819431d5

Johnson, N. A., Jahng, G. H., Weiner, M. W., Miller, B. L., Chui, H. C., Jagust, W. J., et al. (2005). Pattern of cerebral hypoperfusion in Alzheimer disease and mild cognitive impairment measured with arterial spin-labeling MR imaging: initial experience. Radiology 234, 851-859. doi: 10.1148/radiol.2343040197

Joshi, A. D., Pontecorvo, M. J., Clark, C. M., Carpenter, A. P., Jennings, D. L., Sadowsky, C. H., et al. (2012). Performance characteristics of amyloid PET with florbetapir F 18 in patients with alzheimer's disease and cognitively normal subjects. J. Nucl. Med. 53, 378-384. doi: 10.2967/jnumed.111.090340

Kantarci, K., Lowe, V., Przybelski, S. A., Weigand, S. D., Senjem, M. L., Ivnik, R. J., et al. (2012). APOE modifies the association between Abeta load and cognition in cognitively normal older adults. Neurology 78, 232-240. doi: 10.1212/WNL. 0b013e31824365ab

Kawas, C. H., Greenia, D. E., Bullain, S. S., Clark, C. M., Pontecorvo, M. J., Joshi, A. D., et al. (2013). Amyloid imaging and cognitive decline in nondemented oldest-old: the 90+ Study. Alzheimers Dement. 9, 199-203. doi: 10.1016/j.jalz. 2012.06.005

Landau, S. M., Breault, C., Joshi, A. D., Pontecorvo, M., Mathis, C. A., Jagust, W. J., et al. (2013). Amyloid-beta imaging with Pittsburgh compound B and florbetapir: comparing radiotracers and quantification methods. J. Nucl. Med. 54, 70-77. doi: 10.2967/jnumed.112.109009

Landau, S. M., Mintun, M. A., Joshi, A. D., Koeppe, R. A., Petersen, R. C., Aisen, P. S., et al. (2012). Amyloid deposition, hypometabolism, and longitudinal cognitive decline. Ann. Neurol. 72, 578-586. doi: 10.1002/ana.23650

Landau, S. M., Thomas, B. A., Thurfjell, L., Schmidt, M., Margolin, R., Mintun, M., et al. (2014). Amyloid PET imaging in Alzheimer's disease: a comparison of three radiotracers. Eur. J. Nucl. Med. Mol. Imaging 41, 1398-1407. doi: 10.1007/ s00259-014-2753-3

Libon, D. J., Bondi, M. W., Price, C. C., Lamar, M., Eppig, J., Wambach, D. M., et al. (2011). Verbal serial list learning in mild cognitive impairment: a profile analysis of interference, forgetting, and errors. J. Int. Neuropsychol. Soc. 17, 905-914. doi: 10.1017/s1355617711000944

Lim, Y. Y., Ellis, K. A., Ames, D., Darby, D., Harrington, K., Martins, R. N., et al. (2013). Abeta amyloid, cognition, and APOE genotype in healthy older adults. Alzheimers Dement. 9, 538-545. doi: 10.1016/j.jalz.2012.07.004

Lim, Y. Y., Ellis, K. A., Pietrzak, R. H., Ames, D., Darby, D., Harrington, K., et al. (2012). Stronger effect of amyloid load than APOE genotype on cognitive decline in healthy older adults. Neurology 79, 1645-1652. doi: 10.1212/WNL. 0b013e31826e9ae6

Luh, W.-M., Wong, E. C., Bandettini, P. A., and Hyde, J. S. (1999). QUIPSS II with thin-slice TI1 periodic saturation: a method for improving accuracy of quantitative perfusion imaging using pulsed arterial spin labeling. Magn. Reson. Med. 41, 1246-1254. doi: 10.1002/(SICI)1522-2594(199906)41:6<1246:: AID-MRM22>3.0.CO;2-N

Mattsson, N., Tosun, D., Insel, P. S., Simonson, A., Jack, C.R. Jr., Beckett, L. A., et al. (2014). Association of brain amyloid-beta with cerebral perfusion and structure in Alzheimer's disease and mild cognitive impairment. Brain 137(Pt 5), 1550-1561. doi: 10.1093/brain/awu043

Mentis, M. J., Alexander, G. E., Krasuski, J., Pietrini, P., Furey, M. L., Schapiro, M. B., et al. (1998). Increasing required neural response to expose abnormal brain function in mild versus moderate or severe Alzheimer's disease: PET study using parametric visual stimulation. Am. J. Psychiatry 155, 785-794. doi: 10.1176/ajp.155.6.785

Michels, L., Warnock, G., Buck, A., Macauda, G., Leh, S. E., Kaelin, A. M., et al. (2016). Arterial spin labeling imaging reveals widespread and Abetaindependent reductions in cerebral blood flow in elderly apolipoprotein epsilon-4 carriers. J. Cereb. Blood Flow Metab. 36, 581-595. doi: 10.1177/ $0271678 \times 15605847$

Mintun, M. A., Larossa, G. N., Sheline, Y. I., Dence, C. S., Lee, S. Y., Mach, R. H., et al. (2006). [11C]PIB in a nondemented population: potential antecedent marker of Alzheimer disease. Neurology 67, 446-452. doi: 10.1212/01.wnl. 0000228230.26044.a4

Mitrushina, M., Satz, P., Chervinsky, A., and D'Elia, L. (1991). Performance of four age groups of normal elderly on the rey Auditory-Verbal Learning Test.
J. Clin. Psychol. 47, 351-357. doi: 10.1002/1097-4679(199105)47:3<351::AIDJCLP2270470305>3.0.CO;2-S

Mormino, E. C., Kluth, J. T., Madison, C. M., Rabinovici, G. D., Baker, S. L., Miller, B. L., et al. (2009). Episodic memory loss is related to hippocampal-mediated beta-amyloid deposition in elderly subjects. Brain 132(Pt 5), 1310-1323. doi: 10.1093/brain/awn320

Nation, D. A., Edmonds, E. C., Bangen, K. J., Delano-Wood, L., Scanlon, B. K., Han, S. D., et al. (2015). Pulse pressure in relation to tau-mediated neurodegeneration, cerebral amyloidosis, and progression to dementia in very old adults. JAMA Neurol. 72, 546-553. doi: 10.1001/jamaneurol.2014.4477

Nation, D. A., Ho, J., and Yew, B. (2016). Older adults taking AT1-Receptor blockers exhibit reduced cerebral amyloid retention. J. Alzheimers. Dis. 50, 779-789. doi: 10.3233/jad-150487

Nettiksimmons, J., DeCarli, C., Landau, S., and Beckett, L. (2014). Biological heterogeneity in ADNI amnestic mild cognitive impairment. Alzheimers Dement. 10, 511.e-521.e. doi: 10.1016/j.jalz.2013.09.003

Pike, K. E., Ellis, K. A., Villemagne, V. L., Good, N., Chetelat, G., Ames, D., et al. (2011). Cognition and beta-amyloid in preclinical Alzheimer's disease: data from the AIBL study. Neuropsychologia 49, 2384-2390. doi: 10.1016/j. neuropsychologia.2011.04.012

Price, J. L., and Morris, J. C. (1999). Tangles and plaques in nondemented aging and "preclinical" Alzheimer's disease. Ann. Neurol. 45, 358-368. doi: 10.1002/ 1531-8249(199903)45:3<358::AID-ANA12>3.0.CO;2-X

Rentz, D. M., Amariglio, R. E., Becker, J. A., Frey, M., Olson, L. E., Frishe, K., et al. (2011). Face-name associative memory performance is related to amyloid burden in normal elderly. Neuropsychologia 49, 2776-2783. doi: 10.1016/j. neuropsychologia.2011.06.006

Resnick, S. M., Sojkova, J., Zhou, Y., An, Y., Ye, W., Holt, D. P., et al. (2010). Longitudinal cognitive decline is associated with fibrillar amyloidbeta measured by [11C]PiB. Neurology 74, 807-815. doi: 10.1212/WNL. 0b013e3181d 3e3e9

Rodrigue, K. M., Kennedy, K. M., Devous, MD Sr, Rieck, J. R., Hebrank, A. C., Diaz-Arrastia, R., et al. (2012). beta-Amyloid burden in healthy aging: regional distribution and cognitive consequences. Neurology 78, 387-395. doi: 10.1212/ WNL.0b013e318245d295

Roher, A. E., Esh, C., Kokjohn, T. A., Kalback, W., Luehrs, D. C., Seward, J. D., et al. (2003). Circle of willis atherosclerosis is a risk factor for sporadic Alzheimer's disease. Arterioscler. Thromb. Vasc. Biol. 23, 2055-2062. doi: 10.1161/01.atv. 0000095973.42032 .44

Rowe, C. C., Ellis, K. A., Rimajova, M., Bourgeat, P., Pike, K. E., Jones, G., et al. (2010). Amyloid imaging results from the Australian Imaging, Biomarkers and Lifestyle (AIBL) study of aging. Neurobiol. Aging 31, 1275-1283. doi: 10.1016/j. neurobiolaging.2010.04.007

Schneider, J. A., Arvanitakis, Z., Bang, W., and Bennett, D. A. (2007). Mixed brain pathologies account for most dementia cases in community-dwelling older persons. Neurology 69, 2197-2204. doi: 10.1212/01.wnl.0000271090.28148.24

Takahashi, H., Ishii, K., Hosokawa, C., Hyodo, T., Kashiwagi, N., Matsuki, M., et al. (2014). Clinical application of 3D arterial spin-labeled brain perfusion imaging for Alzheimer disease: comparison with brain perfusion SPECT. Am. J. Neuroradiol. 35, 906-911. doi: 10.3174/ajnr.A3780

Telischak, N. A., Detre, J. A., and Zaharchuk, G. (2015). Arterial spin labeling MRI: clinical applications in the brain. J. Magn. Reson. Imaging 41, 1165-1180. doi: 10.1002/jmri.24751

Villemagne, V. L., Pike, K. E., Chetelat, G., Ellis, K. A., Mulligan, R. S., Bourgeat, P., et al. (2011). Longitudinal assessment of Abeta and cognition in aging and Alzheimer disease. Ann. Neurol. 69, 181-192. doi: 10.1002/ana.22248

Wierenga, C. E., Dev, S. I., Shin, D. D., Clark, L. R., Bangen, K. J., Jak, A. J., et al. (2012). Effect of mild cognitive impairment and APOE genotype on resting cerebral blood flow and its association with cognition. J. Cereb. Blood Flow Metab. 32, 1589-1599. doi: 10.1038/jcbfm.2012.58

Wierenga, C. E., Hays, C. C., and Zlatar, Z. Z. (2014). Cerebral blood flow measured by arterial spin labeling MRI as a preclinical marker of Alzheimer's disease. J. Alzheimers. Dis. 42(Suppl. 4), S411-S419. doi: 10.3233/jad-141467

Xekardaki, A., Rodriguez, C., Montandon, M. L., Toma, S., Tombeur, E., Herrmann, F. R., et al. (2015). Arterial spin labeling may contribute to the prediction of cognitive deterioration in healthy elderly individuals. Radiology 274, 490-499. doi: 10.1148/radiol.14140680 
Yokota, M., Saido, T. C., Tani, E., Yamaura, I., and Minami, N. (1996). Cytotoxic fragment of amyloid precursor protein accumulates in hippocampus after global forebrain ischemia. J. Cereb. Blood Flow Metab. 16, 1219-1223. doi: 10.1097/00004647-199611000-00016

Zhang, F., Eckman, C., Younkin, S., Hsiao, K. K., and Iadecola, C. (1997). Increased susceptibility to ischemic brain damage in transgenic mice overexpressing the amyloid precursor protein. J. Neurosci. 17, 7655-7661.

Zlatar, Z. Z., Wierenga, C. E., Bangen, K. J., Liu, T. T., and Jak, A. J. (2014). Increased hippocampal blood flow in sedentary older adults at genetic risk for Alzheimer's disease. J. Alzheimers Dis. 41, 809-817. doi: 10.3233/jad-132252

Zlokovic, B. V. (2011). Neurovascular pathways to neurodegeneration in Alzheimer's disease and other disorders. Nat. Rev. Neurosci. 12, 723-738. doi: $10.1038 / \mathrm{nrn} 3114$
Conflict of Interest Statement: The authors declare that the research was conducted in the absence of any commercial or financial relationships that could be construed as a potential conflict of interest.

Copyright (c) 2017 Bangen, Clark, Edmonds, Evangelista, Werhane, Thomas, Locano, Tran, Zlatar, Nation, Bondi, and Delano-Wood for the Alzheimer's Disease Neuroimaging Initiative. This is an open-access article distributed under the terms of the Creative Commons Attribution License (CC BY). The use, distribution or reproduction in other forums is permitted, provided the original author(s) or licensor are credited and that the original publication in this journal is cited, in accordance with accepted academic practice. No use, distribution or reproduction is permitted which does not comply with these terms. 